\title{
ÄGYPTEN UND DER URSPRUNG DER ESOTERIK. ZUR GESCHICHTE UND GENESE EINES MYTHOS
}

\author{
Jan Assmann
}

Das Alte Ägypten gilt im kulturellen Gedächtnis des Abendlandes als Ursprung und Inbegriff der Esoterik. Das ist die Tradition, deren Konstruktion und Funktion ich nachgehen möchte. Welche Züge der altägyptischen Kultur haben Anlass gegeben zur Entstehung dieser Tradition? Und welche Bedeutung hatte der Mythos des esoterischen Ägypten für das Abendland?

Ägypten war aus dem Kulturgedächtnis des Abendlandes nie verschwunden. Es war durch Antike, Mittelalter und frühe Neuzeit präsent geblieben vor allem als das Land der Hieroglyphen, der Sphinx und der Pyramiden. Mit allen drei dieser kulturellen Markenzeichen verband sich die Vorstellung des Rätsels. Die Pyramiden galten als Weltwunder. Wie und wozu sie errichtet wurden war rätselhaft. Die Sphinx ist der Inbegriff des Rätsels überhaupt. Die Hieroglyphen galten als Geheimschrift zur Überlieferung streng geheimer Mysterien. Die im 19. Jh. mit der Entzifferung der Hieroglyphen entstehende Ägyptologie hat das alte Ägypten nicht entdeckt, sondern nur entzaubert. Die Ägyptologie ist angetreten mit dem Programm der Entmythisierung und Entzauberung der altägyptischen Kultur und steht schon von daher der in Theosophie, Anthroposophie und vielen anderen okkulten Richtungen fortdauernden esoterischen Tradition nicht gerade mit Sympathie gegenüber. Für die zünftige Ägyptologie handelt es sich hierbei schlichtweg um ein Mißverständnis. Die Griechen haben ihre Mysterien und ihre platonisierende Mystik in die altägyptische Kultur hineinprojiziert, und da das Abendland alle seine Informationen über diese Kultur aus griechischen, lateinischen, hebräischen und arabischen Quellen bezog, ist sie diesem Mißverständnis aufgesessen. Das esoterische Ägypten, das ist das Fazit selbst noch von Erik Hornungs mit so viel Empathie und Verständnis geschriebenem Buch, ist eine Konstruktion der Rezeptionsgeschichte, der auf Seiten der altägyptischen Kultur selbst so gut wie nichts entspricht. Immerhin hat aber die Ägyptologie seit einigen Jahren ein ganz neues Verhältnis zu dieser Rezeptionsgeschichte entwickelt, und Hornungs Buch ist ein Pionier 
dieser Wende. ${ }^{1}$ Während wir sie früher als ein Kuriosum im Museum der Mißverständnisse belächelten, haben wir gelernt, sie als ein Faktum unseres kulturellen Gedächtnisses ernst zu nehmen und gehen sogar einen Schritt weiter, indem wir fragen, ob uns diese Rezeptionsgeschichte nicht doch auch wichtige Aufschlüsse über die ägyptische Kultur selbst vermittelt.

Beginnen wir mit der Idee der Geheimniskultur, die in der Spätantike recht verbreitet ist. Hier stoßen wir bei verschiedenen Autoren auf die Vorstellung, daß alle Religionen, insbesondere aber die altägyptische, doppelbödig sind, daß sie eine Außenseite haben, die sich jedem Gläubigen und jedem Reisenden darbietet, und eine Innenseite, von der nur die Eingeweihten und Weisen Kenntnis haben. Die wichtigste Stelle steht bei Origenes in seiner Schrift Contra Celsum. Celsus hatte in seiner Streitschrift gegen die Christen (alethes logos) behauptet, alles, was zum Christentum gehört, durch und durch zu verstehen. Origenes meint dazu:

Celsus scheint sich mir hier zu verhalten wie jemand, der nach Ägypten reist, wo die weisen Ägypter entsprechend ihrer landesüblichen Gelehrsamkeit viel philosophieren über jene Dinge, die sie als göttlich betrachten, während die Einfältigen derweil nur bestimmte Fabeln hören, die ihnen viel Vergnügen bereiten. Celsus, sage ich, verhält sich wie ein Ägyptenreisender, der sich nur mit jenen Einfältigen unterhalten hat und von keinem der Priester das Geringste erfahren hat über ihre geheimen und höheren Mysterien, und der nun prahlt, daß er alles kennt, was zur ägyptischen Theologie gehört...W Was wir jetzt festgestellt haben in bezug auf den Unterschied zwischen Weisen und Einfältigen unter den Ägyptern, das gilt auch für die Perser, unter denen die religiösen Riten von den Einsichtigen auf sinnvolle Weise ausgeübt werden, während die oberflächliche Menge nicht weiter blickt als auf das äußere Symbol oder Zeremonial. Und dasselbe gilt gleicherweise für die Syrer und Inder und alle anderen Völker, die neben ihren religiösen Fabeln eine Wissenschaft und Lehre besitzen. ${ }^{2}$

Mit diesen Sätzen sagt Origenes zweierlei. Er stellt erstens fest, daß alle ihm bekannten Religionen eine esoterische Dimension besitzen, die nur den Weisen zugänglich ist, und behauptet zweitens, daß dasselbe auch für das Christentum gilt, so daß ein Außenstehender wie Celsus davon nur allenfalls die exoterische Außenseite zu Gesicht bekommt.

${ }^{1}$ Hornung, Das esoterische Ägypten.

2 Origenes, 'Contra Celsum' 677-78. 
Für Clemens von Alexandrien bestand in dieser Hinsicht sogar kein Unterschied zwischen der ägyptischen und der israelitischen Religion:

Die Ägypter bezeichneten den wirklich geheimen Logos, den sie im innersten Heiligtum der Wahrheit bewahrten, durch „Adyta“, und die Hebräer [bezeichneten ihn] durch den Vorhang [im Tempel]. Was daher die Verheimlichung angeht, sind die Geheimnisse [ainigmata] der Hebräer und der Ägypter einander sehr ähnlich. ${ }^{3}$

Auch Eusebius zählt die alttestamentliche Religion zu den Geheimnisreligionen. Er vertrat die Ansicht, daß auch die Tora ein doppelbödiger Text ist, der den Einfältigen eine Menge konkreter Vorschriften macht, den Weisen aber eine noch viel größere Menge zu denken gibt.

Moses befahl dem jüdischen Volk, alle Riten auf sich zu nehmen, die in den Worten ihres Gesetzes beschlossen waren. Aber er wollte auch, daß die anderen, deren Geist und Tugend stärker waren weil sie frei waren von dieser äußeren Schale, sich gewöhnen sollten an eine göttlichere, über die Fassungskraft des gemeinen Mannes hinausgehende Philosophie und mit den Augen des Geistes in den höheren Sinn der Gesetze eindringen sollten. ${ }^{4}$

Im alten Ägypten aber, darin war man sich einig, sah man den Ursprung und das Modell der Geheimnisreligion.

Über die Geheimnisse der Ägypter äußert sich Clemens an vielen anderen Stellen, darunter an folgender, die die Exklusivität der Einweihung betont:

Die Ägypter offenbaren ihre religiösen Mysterien nicht unterschiedslos allen, noch teilen sie das Wissen um die göttlichen Dinge den Profanen mit, sondern nur denen, die zur Nachfolge im Königtum ausersehen sind und zu solchen von den Priestern, die dazu aufgrund ihrer Geburt und Erziehung am besten qualifiziert sind. ${ }^{5}$

Also gehören nicht nur Weisheit, sondern auch Herrschaft und Geheimnis zusammen. Das Geheimnis, die esoterische Seite, der sensus mysticus der Religion, hat eine politische Funktion. Davon weiß auch Plutarch. In seiner Schrift über Isis und Osiris schreibt er

${ }^{3}$ Das Zitat nach Reinhold, Die hebräischen Mysterien, 55, ist eine Kombination von Clemens von Alexandriens stromata Buch V: cap. III, 19.3 und cap. VI, 41.2. Siehe Alexandrinus, Stromata Buch $I-V I, 338$ und 354.

${ }^{4}$ Auch dieses Zitat nach Reinhold, a.a.O. 88. Es handelt sich um Eusebius, 'Praeparatio Evangelica' 454.

${ }^{5}$ Alexandrinus, Stromata V, cap. vii. 41.1. 
Wenn unter den Ägyptern ein König aus dem Militärstand gewählt wird, bringt man ihn von Stund an zu den Priestern und unterrichtet ihn in jener Arkantheologie, die geheimnisvolle Wahrheiten unter obskuren Fabeln und Allegorien verbirgt. Daher stellen sie Sphingen vor ihren Tempeln auf um zu bedeuten, daß ihre Theologie eine gewisse arkane und rätselvolle Weisheit enthält. ${ }^{6}$

Den Eingeweihten, Priestern und Herrschern, wird strengste Verschwiegenheit auferlegt.

Deren Symbol ist der Gott Harpokrates, der als Kind mit dem Finger am Mund dargestellt wird. Hierzu Plutarch:

Den Harpokrates der Ägypter darf man nicht für einen unvollkommen und kindlichen Gott halten, sondern für den, der über die Rede der Menschen bezüglich der Götter gesetzt ist, die nur unvollkommen, stammelnd und unartikuliert ist, und zugleich der, der sie reguliert und korrigiert; wobei der Finger an seinem Mund ein Symbol für Schweigen und Verschwiegenheit ist. ${ }^{7}$

In den alten Religionen, das war weitverbreitete oder sogar allgemeine Überzeugung, hat Alles einen Hintersinn. Hinter jeder offiziellen Religion gibt es eine geheime Überlieferung, eine „Kabbala“. Jede Überlieferung ist doppelbödig. Das Wissen ist nun einmal ungleich verteilt in der Welt und die Wahrheit ist ohnehin nur verhüllt zu haben. Dahinter steht die mittel- und neuplatonische Erkenntnistheorie, derzufolge die Wahrheit in dieser Welt immer nur annäherungsweise, in Rätseln, Fabeln und Allegorien anzudeuten, aber nie unmittelbar zu erkennen und darzustellen sei. Die phänomenale Welt ist der Schleier, in dem sich die Wahrheit zugleich offenbart und verhüllt. Die Wahrheit ist schwer zugänglich, und nur der Weise kann es wagen, sich ihr wenigstens anzunähern. Die antike Theorie der ägyptischen Geheimreligion, wie wir sie bei Plutarch und Diodor, Philo, Origenes und Clemens von Alexandrien sowie bei Porphyrios und Jamblich antreffen, geht von dem Prinzip aus, daß die Wahrheit an sich ein Geheimnis darstellt und in dieser Welt nur verschleiert in Bildern, Mythen, Allegorien

${ }^{6}$ Plutarch, De Iside, 354 (cap. 8). Cf. Alexandrinus., Stromata .V, cap. v. 31.5: ,Daher stellen die Ägypter Sphingen vor ihre Tempel, um dadurch anzuzeigen, daß ihre Gotteslehre rätselvoll und dunkel ist... Aber vielleicht liegt die Bedeutung jener ägyptischen Sphingen auch darin, anzudeuten, daß die Gottheit sowohl geliebt als auch gefürchtet werden will; geliebt als gütig und dem heiligen Menschen wohlgesonnen, aber gefürchtet als unerbittlich gerecht gegen den Gottlosen, da die Sphinx aus dem Bild eines Menschen und eines Löwen zusammengesetzt ist'.

${ }^{7}$ Plutarch, De Iside, cap. 68. 
und Rätseln zu fassen ist. Am schönsten kommt diese im Neuplatonismus, Hermetismus und in der Gnosis verbreitete Anschauung in dem Philippus-Evangelium zum Ausdruck, einem in koptischer Sprache überlieferten gnostischen Text aus Ägypten:

Die Wahrheit kam nicht nackt in die Welt, sondern sie kam in den Sinnbildern und Abbildern.

Die Welt wird sie auf keine andere Weise erhalten. ${ }^{8}$

So wie die Worte „Ich bin alles, was war, ist und sein wird“ auf der Inschrift des Bildes zu Sais sich als die Verweigerung eines Namens, aber auch als die Offenbarung der namenlosen Gottheit deuten lassen, so bedeutet der Schleier in den folgenden Worten-, "meinen Schleier hat kein Sterblicher aufgedeckt" - sowohl ein Verbergen, als auch ein zur Erscheinung kommen der Gottheit. Goethe hat diesen Doppelaspekt des Geheimnisses als Verhüllung und als Manifestation der Wahrheit sehr prägnant formuliert:

Das Wahre ist gottähnlich; es erscheint nicht unmittelbar, wir müssen es aus seinen Manifestationen erraten. ${ }^{9}$

Wodurch kam das Alte Ägypten in den Ruf einer paradigmatischen Geheimniskultur? Hierfür sind vor allem zwei Phänomene verantwortlich, die zunächst die Antike und, von ihrem Erbe ausgehend, dann das spätere Abendland ganz besonders nachhaltig fasziniert haben. Das eine ist die Schrift, das andere die Architektur. Beides hat man als Hinweis aufgefaßt auf eine doppelte, in Exoterik und Esoterik gespaltene Kultur. In beiden Fällen beruht die Faszination der Antike auf einem Mißverständnis, das aber in der abendländischen Tradition im höchstem Maße geschichtsmächtig geworden ist und unsere geistige Welt ganz entscheidend mitgeprägt hat.

Beginnen wir mit der Schrift. Die Faszination der Griechen für die ägyptische Schriftkultur beruht gleich auf einer ganzen Reihe von Mißverständnissen: daß die Ägypter nicht ein, sondern zwei oder sogar drei ganz verschiedene Schriftsysteme nebeneinander benutzten, daß diese Schriften für verschiedene Zwecke verwendet wurden, daß die Hieroglyphen innerhalb dieses polygraphischen Systems eine Geheimschrift darstellten und daß sie sich nicht auf Laute, sondern auf Dinge

${ }^{8}$ Foerster (Hg.), ,Philippus-Evangelium', 108.

${ }^{9}$ Goethe, ,Aus Makariens Archiv', 460 Nr.3. 
und Begriffe bezögen. Nichts davon stimmt, aber so ganz falsch ist es andererseits auch wieder nicht. ${ }^{10}$

Tatsächlich benutzten die Ägypter, als die Griechen mit ihnen in Berührung kamen, drei Schriften, Hieroglyphen, Hieratisch und Demotisch, die äußerlich sehr verschieden aussahen, auch wenn sie aus derselben Wurzel entwickelt waren. Es stimmt auch, daß diese Schriften für verschiedene Zwecke verwendet wurden. Was die Griechen daran besonders faszinierte, war die vermeintliche Verwendung zweier oder dreier ganz verschiedener Schriftsysteme: einer Bilderschrift und einer abstrakten Lautschrift. Mit der Frage, ob es sich um zwei oder drei verschiedene Schriften handelt, wollen wir uns nicht lange aufhalten. Wer zwei Schriften zählt, unterscheidet zwischen Hieroglyphen und Kursivschrift, wer drei zählt, unterscheidet innerhalb der Kursivschriften noch einmal zwischen Klassisch-Hieratisch und Spät-Demotisch. Heute wissen wir, daß es sich hier lediglich um drei Formen eines einzigen Schriftsystems-Monumentalschrift („Hieroglyphen“) und Handschrift („Hieratisch“ bzw. „Demotisch“)-handelte; die Griechen aber deuteten sich den Befund so, daß die Handschrift für Alle („Demotisch“, von gr. demos „Volk“ heißt ja „Volksschrift"), die Monumentalschrift aber nur für die Priester da war. So schrieb etwa Diodor:

Die Ägypter besitzen nämlich zwei Schriften: die eine, "demotisch“ genannt, lernen alle; die andere wird die "heilige“ genannt. Bei den Ägyptern verstehen sie allein die Priester, die sie von den Vätern in den Mysterien lernen. ${ }^{11}$

Die Hieroglyphen galten Diodor also als eine Mysterienschrift. Diodor wiederum fußt mit seinen Informationen über die altägyptische Kultur auf einem verlorenen Werk des Hekataios von Abdera, der Ende des 4. Jhs. v.Chr. in Alexandria lebte.

In seinen ,Teppichen', jenem groß angelegten Werk, das heidnischantike und biblisch-christliche Überlieferungen $\mathrm{zu}$ einem neuen Konzept christlicher Bildung vereinigen sollte, schreibt Clemens von Alexandrien um das Jahr $200 \mathrm{n}$. Chr. über die Ausbildung eines ägyptischen Schriftgelehrten:

\footnotetext{
${ }^{10}$ Siehe hierzu die Beiträge Assmann (Hg.), ,Hieroglyphen', 'Antike Äußerungen zur ägyptischen Schrift', ,Etymographie‘.

${ }^{11}$ S. ,Antike Äußerungen' (n. 11), $33 \mathrm{f}$.
} 
Diejenigen, die bei den Ägyptern unterrichtet werden, lernen zuerst von allem die Verwendungsart der ägyptischen Schrift, die den Namen Briefschrift [= demotisch] führt, als zweite sodann die hieratische, die von den heiligen Schreibern (Hierogrammaten) angewendet wird, zuletzt und als höchste Stufe der Einweihung die Hieroglyphenschrift, die teils „vermittels der elementaren Buchstaben“ (dia ton proton stoicheion) etwas unmittelbar benennt (kyriologeisthai), teils symbolisch ist. Von der symbolischen Schrift gibt es drei Arten: die eine bezeichnet etwas unmittelbar (kyriologeisthai), indem sie seine Form im Bilde wiedergibt (kata mimesin), die andere wird in der Weise geschrieben, dass ein Zeichen auf ein anderes Gebiet übertragen wird (tropikos), die dritte schließlich verfährt allegorisch und gibt gewissermaßen Rätsel auf (kata tinas ainigmous). ${ }^{12}$

Aus diesen Nachrichten zur ägyptischen Schriftkultur reimte sich das 17. Jahrhundert die Idee einer „doppelten Religion " mit der allgemein zugänglichen Außenseite einer polytheistischen Volksreligion und der nur den Eingeweihten zugänglichen Innenseite eines philosophischen Monotheismus zusammen. Während das Volk den vielen Göttern huldigte, von denen man glaubte, daß sie belohnend und strafend über die Einhaltung der Gesetze wachten und das Staatswesen nach innen und außen schützten, widmeten sich die Eingeweihten der verborgenen, All-Einen Gottheit, auf deren Sitzbild im Tempel von Sais, Plutarch zufolge, geschrieben stand: „Ich bin alles, was da war, ist und sein wird. Kein Sterblicher hat meinen Schleier gehoben. ${ }^{\text {"13 }}$

Die andere Eigentümlichkeit der ägyptischen Kultur, die zur Vorstellung einer doppelten, in Exoterik und Esoterik gespaltenen Kultur führte, ist die Architektur. Diese Deutung hängt eigentümlicherweise mit der Sintflut und anderen Vorstellungen von Flut- und Feuerkatastrophen zusammen. Hier geht es um die Vorstellung von der Verwundbarkeit des Wissens und der Kultur überhaupt, und um das Problem, wie ihrem Untergang vorzubeugen sei. Im Zentrum dieser Überlieferung steht die Idee, dass die Fülle des Wissens nicht am Ende sondern am Anfang der Menschheitsgeschichte steht. Adam, der die Tiere benennen konnte, war im Besitz des ursprünglichen Schöpfungswissens. Mit der Sintflut ging dieses Wissen verloren, das sich von Adam auf seine Nachkommen vererbt hatte. ${ }^{14}$

\footnotetext{
12 Stromata, V, IV.

13 Plutarch, De Iside, cap. 9, $354 \mathrm{C}$.

${ }^{14}$ S. hierzu Assmann (Hg.), Sintflut und Gedächtnis.
} 
Interessant ist diese Überlieferung vor allem deswegen, weil sie mit der Vorstellung verbunden ist, ein Rest dieses Urwissens sei gleichwohl gerettet worden. Genau wie durch Noah und seine Arche ein Rest des Lebens auf dieser Erde über die Sintflut hinweg gerettet werden konnte, so ist auch durch schriftliche Aufzeichnung ein Rest des Urwissens erhalten geblieben. In der jüdischen und christlichen Überlieferung verbindet sich diese Idee mit der Vorstellung zweier Pfeiler oder Stelen, auf denen Adam oder seine Nachkommen dieses Urwissen inschriftlich kodifiziert hätten. Am bekanntesten ist die schon von Josephus Flavius berichtete frühjüdische Legende, die diese Kodifizierung vorsintflutlicher Urweisheit mit den Kindern des Seth verbindet. ${ }^{15}$ Diese gelten als die Erfinder der Astronomie, des himmlischen Wissens von den Gestirnsbewegungen. Eingedenk der Weissagung Adams, dass die Welt durch Feuer- und Wasserkatastrophen untergehen würde, beschliessen sie, ihr Wissen auf zwei Pfeilern niederzuschreiben, einem aus Ziegeln für die Feuer- und einem aus Stein für die Wasserkatastrophe. ${ }^{16}$,Der steinerne Pfeiler, setzt Josephus hinzu, ,steht übrigens noch heute in Syrien. ${ }^{17}$ Offenbar handelt es sich um eine Legende, die sich an ein mit unlesbaren Schriftzeichen, vermutlich ägyptischen oder hethitischen Hieroglyphen, bedecktes Monument geknüpft hat.

Ganz ähnliche Vorstellungen begegnen nun aber auch ausserhalb der jüdisch-christlichen Überlieferung, vor allem im Umkreis der hermetischen Tradition, wo sie eine ganz besonders prominente Rolle spielten. Bereits eine bei Synkellos überlieferte, von ihm dem Manetho (1. Hälfte 3.Jh.v.Chr.) zugeschriebene, aber sicher aus späterer Zeit stammende Passage handelt von dem über eine Flutkatastrophe hinweg geretteten Urwissen:

Er (Manetho) war ein Hohepriester in der Zeit des Ptolemaeus Philadelphos und schrieb auf der Grundlage von Inschriften im siriadischen Lande (Ägypten), ${ }^{18}$ die von Thoth, dem ersten Hermes, in heiliger Sprache und Schrift aufgezeichnet waren. Nach der Flut habe sie der zweite Hermes, Sohn des Agathodaimon, in Hieroglyphen übertragen und in den ägyptischen Tempeln in Büchern niedergelegt. ${ }^{19}$

${ }^{15}$ Flavius Josephus, Antiquitates, Kap.2; Ginzberg, Legends of the Jews, 121f.

${ }^{16}$ Eine Darstellung des Seth mit den beiden Pfeilern findet sich z.B. in Buno, Historische Bilder auf der Bildtafel „Das 1. TausendJahr: Die Patriarchen vor der Sündflut“. Ich verdanke die Kenntnis dieser Darstellung Gerhard F. Strasser.

${ }^{17}$ Josephus, a.a.O.

18 "siriadisch", von „Sirius“ = Süden, Ägypten.

19 Waddell, Manetho, Appendix I, 208f. 
Besonders einflußreich ist aber eine Stelle bei Ammianus Marcellinus geworden, die sich nun explizit auf die Architektur, und zwar auf die beschrifteten unterirdischen Grabanlagen der Ägypter, und im besonderen die Königsgräber im Tal der Könige zu Theben bezieht. Er schreibt:

sunt et syringes subterranei quidam et flexuosi secessus, quos, ut fertur, periti rituum vetustorum, adventare diluvium praescii, metuentesque ne caerimoniarum oblitteraretur memoria, penitus operosis digestos fodinis, per loca diversa struxerunt, et excisis parietibus, volucrum ferarumque genera multa sculpserunt et animalium species innumeras multas, quas hierographicas litteras appellarunt.

Es gibt auch Syringen, das heißt unterirdische und gewundene Gänge. Der Überlieferung zufolge ließen die in die alten Riten Eingeweihten sie an verschiedenen Orten mit ungeheurem Aufwand aushauen, da sie die Heraufkunft einer Flutkatastrophe voraussahen und fürchteten, die Zeremonien könnten in Vergessenheit geraten. Auf die dergestalt aus dem Felsen geschlagenen Wände ließen sie alle möglichen Arten von Vögeln und Tieren einmeißeln: das nennen sie „Hieroglyphen“. ${ }^{20}$

Diesem Motiv des über die Sintflut hinweg geretteten vorsintflutlichen Wissens begegnen wir vor allem auch im Umkreis der hermetischen Tradition, wo es eine ganz besonders prominente Rolle spielte. Arabische mittelalterliche Autoren, insbesondere Ibn Abi Usaybia setzen die Enkel Adams mit dem "ersten Hermes“ (Irmis/Idris) gleich; er habe sein Wissen nicht auf Pfeiler, sondern auf Tempelwände geschrieben. ${ }^{21}$ Idrisi überträgt sogar das Motiv von Stein und Ziegel auf den Tempelbau. Weil Hermes nicht genau gewusst habe, ob die Welt durch das Feuer oder durch das Wasser vernichtet werde, habe er, um das Wissen zu schützen, sowohl irdene als auch steinerne Tempel bauen lassen, da er glaubte, die ersten könnten dem Feuer, die zweiten dem Wasser Widerstand leisten. Unter diesen Tempeln wird der von Achmim

${ }^{20}$ Ammianus Marcellinus XXII, 15.30 = Marcellin, Histoire, 140 .

${ }^{21}$ F. Ebeling, Diss. \$5.1. Der erste (Hermes) sei der Enkel Adams gewesen und habe vor der Sintflut gelebt. Er sei von den Hebräern mit Henoch und von den Arabern mit Idris identifiziert worden. Von Adam selbst sei er unterrichtet worden. Als erster habe er Astronomie betrieben, Tempel gebaut, um Gott darin anzubeten, habe die Medizin studiert und darüber geschrieben. Er (und nicht Adam) habe vorausgesehen, dass die Welt durch Wasser und Feuer zerstört werde, und vor der Sintflut gewarnt. Um die Wissenschaft vor dem Untergang zu retten, habe dieser in Oberägypten lebende Hermes neben Pyramiden auch den Tempel von Achmim errichtet, in dem all seine Wissenschaft in Stein eingemeißelt worden sei. 
herausgehoben..$^{22}$ Durch diese Rettungsaktion ist das Urwissen mit der Sintflut nicht verloren gegangen, sondern nur okkultiert: es wurde zum Geheimwissen weniger Eingeweihter, die die antediluvianische Schrift zu entziffern und das in ihr gespeicherte Wissen um die Geheimnisse der Schöpfung zu deuten und weiterzugeben wußten. Die hieroglyphischen Inschriften in den ägyptischen Tempeln und unterirdischen Grabanlagen verstand man als Kodifikationen hermetischen Wissens, das vor der vorausgesehenen Katastrophe gerettet werden sollte. Ibn Abi Usaybia deutete insbesondere einen Tempel in Achmim als hermetischen Wissensspeicher; Achmim war in der Spätantike eine Hochburg ägyptischer Tradition. Achmim war der Sitz einer gelehrten Familie, zu der u.A. der Dichter Nonnos und der Gelehrte Horapollon, der Verfasser des berühmten Hieroglyphenbuchs gehörten. ${ }^{23}$ Kein Wunder, dass sich gerade mit dieser Stadt das Motiv des geretteten Wissens verband. Übrigens versteht man in der Tat den Typus des spätägyptischen Tempels mit seinen von unten bis oben mit Inschriften und Bildern bedeckten Wänden, Pfeilern und Durchgängen als Aufzeichnungsform und Speicher des religiösen Wissens, das man von Vergessen bedroht glaubte angesichts zwar nicht einer Flutkatastrophe, aber des Hellenismus. ${ }^{24}$ In der arabischen Überlieferung erscheint die Sintflut offensichtlich als eine Chiffre für den Gedächtnisverlust, den der Untergang der ägyptischen Kultur und der Kenntnis der Hieroglyphen bedeutete. Dass eine gelehrte Elite, die im Ägypten des arabischen Mittelalters unter den unzähligen mit Hieroglyphen bedeckten Monumenten der altägyptischen Kultur lebte, auf den Gedanken kommen konnte, mit den Aufzeichnungen eines vergessenen Wissensschatzes konfrontiert zu sein und die Gründe für dieses Vergessen in der Sintflut suchte, das erscheint durchaus plausibel.

Die Sintflut wurde auf diese Weise zur Geburt der Esoterik. Sie sorgte für einen allgemeinen Gedächtnisverlust und machte das Wissen exklusiv. Aus dem Gemeinwissen der Menschheit wurde das Geheimwissen der Adepten. Diese Überlieferung verbindet sich vor allem mit

${ }^{22}$ Dozy und de Goeje, Description de l'Afrique, 46ff; Edrisi oder Idrisi war dem Abendland kein Unbekannter. Seine Geographia Nubiensis wurde 1591 in Rom gedruckt. In der Übersetzung von Gabriele Sionita unter dem Titel: Geographia Nubiensis: id est accuratissim, a totius orbis in septem climata divisi descriptio [...] recens ex Arabico in latinam versa 1619. Ich verdanke diese Hinweise F. Ebeling.

${ }_{23}$ Thissen, Horapollon.

${ }^{24}$ S. hierzu meinen Beitrag ,Das gerettete Wissen. Flutkatastrophen und geheime Archive', 291-301. 
Hermes Trismegistos: er gilt als der grosse Interpret und Transkodifikator dieses Urwissens, das in komprimierter hieroglyphischer Form auf den hermetischen Stelen in einer Höhle in Ägypten aufgezeichnet ist und von Hermes in unzähligen, teilweise auch ins Griechische übersetzen Büchern dekomprimiert und verbreitet wurde..$^{25}$

Was nun seit dem 17. und 18. Jahrhundert die Reisenden und Antiquare wie Richard Pococke, Ludwig Frederik Norden, Athanasius Kircher und Bernard de Montfaucon der westlichen Gesellschaft in gelehrten Bildwerken über Ägypten berichteten, schien den Befund einer doppelten Religion aufs Glänzendste zu bestätigen. Ganz Ägypten, so konnte man lesen und auf Bildtafeln sehen, war unterminiert von unterirdischen Hallen, Kammern und Gängen, die über und über mit den priesterlichen Hieroglyphen bedeckt waren, und die nur einem Zweck dienen konnten: der Geheimreligion der Eingeweihten als Kultbühne, Forschungsstätte und Wissensspeicher zu dienen. Besonders beliebt war diese Überlieferung über das in den unterirdischen Anlagen der Ägypter gerettete prädiluviale Urwissen bei den Freimaurern des 18. Jhs., die sich als die Erben und Fortsetzer dieser esoterischen Tradition fühlten. Anton Kreil, ein Philosoph, Philologe und als Freimaurer und Illuminat Mitglied der Loge zur Wahren Eintracht in Wien geht darauf in einem Logenvortrag über die ,Wissenschaftliche Freimaurerei' ein, den er in zwei Sitzungen im April 1785 vorgetragen hatte. Der Vortrag ist auch deswegen interessant, weil bei diesen Sitzungen Leopold Mozart zum Gesellen und Meister erhoben wurde, und auch W.A. Mozart dabei anwesend war. ${ }^{26}$ Der ,ägyptische Priesterorden', schreibt Kreil, scheint nach allem, was sich ,aus den historischen Überbleibseln schließen läßt, in dem Besitze der gesammelten Kenntnisse der Vorwelt gewesen zu seyn. 'Strabo zufolge schrieben die Priester, alles, was höhere und feinere Gelehrsamkeit war, in ihren geheimen Schriften' auf und gaben nur einen Teil davon an Platon und Eudoxos, die 13 Jahre bei ihnen zubrachten, weiter. Diese Priester, und nun folgt eine schier unglaubliche Theorie über die Formen altägyptischer Wissenskultur, ,haben die Hälfte ihres Lebens in unterirdischen Höhlen zugebracht', sie hatten ,eine sonderbare Leidenschaft für das Aushöhlen der Felsen' und bauten

${ }^{25}$ Anonymus, Athenian 95-100 (Brief XXV des Orsames, von Theben).

${ }^{26}$ Kreil, Über die wissenschaftliche Maurerey 49-78. Dazu und zu Mozarts Anwesenheit siehe mein Buch Die Zauberflöte, 100-106. 
übrigens für die Unvergänglichkeit. 160 Fuß unter den Pyramiden waren Gemächer, welche miteinander durch Gänge kommunizierten, die Ammianus Marcellus auf griechisch syringes nennt.... Kurz, alles war mit Grotten, Höhlen und unterirdischen Gängen besetzt und durchschnitten. ${ }^{27}$

Kreil zitiert aus einem zeitgenössischen Werk:

Täglich entdecken die Reisenden derer mehrere; denn itzt hat man noch kaum den hundertsten Teil davon entdecket. Wenn man diese Art, unter der Erde zu studieren, betrachtet, so dürfen wir uns nicht wundern, daß die Priester dadurch sichs zur Gewohnheit gemacht haben, alle ihre wahre oder vermeintliche Wissenschaft unter einem beynahe undurchdringlichen Schleyer zu verhüllen. ${ }^{28}$

Eine phantastischere Deutung haben die ägyptischen Königsgräber (die noch Champollion auf Grund der Stelle aus Ammianus Marcellinus als „syringes" bezeichnete) und sonstigen über und über beschrifteten ägyptischen Grabanlagen wohl kaum je erfahren. Dazu muß man wissen, daß diese „syringes“, die Kreil und sein Gewährsmann de Pauw ,160 Fuß unter die Pyramiden'verlegen, vielmehr über 600 Kilometer südlich der Pyramiden von Giza im Tal der Könige zu Theben liegen. Sie gelten Kreil und de Pauw nicht nur als Wissensspeicher, sondern auch als Versammlungs- und Studienorte. Die Technik, „unter der Erde zu studieren", hielten sie für eine Strategie der Geheimhaltung. Kreil mag auch an eine Stelle bei Lukian gedacht haben, wo von einem ägyptischen Weisen die Rede ist, der ganze 23 Jahre unter der Erde studiert haben soll:

Auf der Rückreise trug es sich zu, daß ein Mann aus Memphis mit uns fuhr, ein Mann von erstaunlicher Weisheit, und ein wahrer Adept in allen Egyptischen Wissenschaften. Man sagte von ihm, er habe ganzer drey und zwanzig Jahre unter der Erde gelebt, und sey während dieser Zeit von der Isis selbst in der Magie unterrichtet worden.

Du sprichst, unterbrach ihn Arignotus, von meinem ehmaligen Lehrer Pankrates? war es nicht ein Mann vom Priester-Orden, mit abgeschornen Haaren, der keine andere als leinene Kleider trug-immer in tiefen Gedanken-sprach sehr rein Griechisch-ein langgestreckter Mann, mit herabhängender Unterlippe, und etwas dünnen Beinen? ${ }^{29}$

${ }^{27}$ Kreil, ,Über die wissenschaftliche Maurerey', $64 f$.

${ }^{28}$ Kreil zitiert aus de Pauw, Recherches philosophiques.

${ }^{29}$ Lukian, Philopseudes, cap. 33. 
,In dieser Absicht', paraphrasiert Kreil die Passage bei Ammianus Marcellinus,

trafen sie alle Menschen mögliche Anstalten, ober und unter der Erde, führten ungeheure Steinmassen auf, denen sie eine uns unerreichbare Unvergänglichkeit zu geben wußten, und gruben ihre Weisheit in Hieroglyphen verkleidet in Pyramiden, Obelisken, steinerne Tafen und Säulen zur stummen Aufbewahrung ein. ${ }^{30}$

Daran ist jedenfalls auch aus heutiger Sicht nicht zu zweifeln, daß die Ägypter in der Errichtung und Beschriftung ihrer zahllosen Monumente von einem beispiellosen Willen zur Überlieferung besessen waren, auch wenn es bei dieser Überlieferung vielleicht nicht um die Art von Kenntnissen ging, an die die Freimaurer anschließen zu können glaubten.

Die ägyptischen Priester kodifizierten nun nicht nur ihr geheimes Wissen in unterirdischen Speichern, sondern, wählten ausserdem noch die rechtschaffensten, geprüftesten und hellsten Köpfe aus, um ihnen, nach gehöriger Ausbildung, Prüfung und Einweihung, das kostbare Pfand ihrer Geheimnisse zur Überlieferung auf die Nachkommenschaft anvertrauen zu können. ${ }^{31}$ Einen anderen Rat hat auch der Semiotiker Th. A. Sebeok nicht gewußt, als er von einer mit der Lagerung radioaktiver Abfallstoffe befassten US-amerikanischen Firma beauftragt wurde, ein Aufzeichnungssystem für Informationen über Lagerungsort und Eigenschaften des Atommülls zu entwickeln, das auch nach 10000 Jahren einer der heutigen Sprachen und Schriftsysteme unkundigen Nachwelt noch irrtumsfrei lesbar wäre. ${ }^{32}$

Sebeoks...Vorschlag lief darauf hinaus, eine ,Atompriesterschaft' zu berufen, eine erste Generation von Physikern, Linguisten, Strahlenexperten und Semiotikern, mit der eine Dynastie begründet werden sollte, die über Generationen hinweg die Botschaft immer wieder neu zu codieren hätte, um auf diese Weise für die Stabilität und sichere Übermittlung der Nachrichten zu sorgen. ${ }^{33}$

Die Parallele ist perfekt. Die Aufgabe, vor die sich Sebeoks ägyptische Vorgänger gestellt sahen, betraf ebenfalls die Entwicklung eines Informationssystems, das bis in fernste Zukunft lesbar bliebe, um das

\footnotetext{
${ }^{30}$ Kreil, ,Über die wissenschaftliche Maurerey', $64 \mathrm{f}$.

${ }^{31}$ Ebd.

${ }^{32}$ Schneider, ,Liturgien der Erinnerung; Assmann, Erinnerungsräume, $352 \mathrm{f}$.

${ }^{33}$ Schneider, ,Liturgien der Erinnerung', 676f.
} 
Geheimwissen der Priester irrtumsfrei zu überliefern, und erforderte dieselben drei Lösungen: (a) die Entwicklung eines sprachunabhängigen Zeichensystems (die Hieroglyphen), (b) die Codierung und Speicherung der Informationen in zeitresistenter Form (die unterirdischen Wissensspeicher) und (c) die Gründung eines elitären Priesterordens, der über Generationen hinweg die Botschaft zu überliefern und neu zu codieren hätte. Kreil jedenfalls schließt

aus der Kunst, der Vorsicht, und dem unermeßlichen Aufwand, wodurch sie den einen Theil ihres Zweckes so meisterlich erreichten, auf die Güte der anderen Hälfte ihres Plans, nämlich auch den lebendigen Geist der Hieroglyphe in verschwiegenen und unsterblichen Mysterien der bessern Nachwelt zu überliefern “ und zieht daraus "den Schluß, daß es nicht vernunftwidrig ist, anzunehmen, daß ihre geheime Weisheit noch in unsern Tagen, so wie ihre Pyramiden, Obelisken und Sphinge, existiere. ${ }^{34}$

Diese Weisheit ist zwar unzugänglich, aber es gibt sie noch, im Sinne eines kulturellen Unbewußten, das auf eine dem Bewußtsein unzugängliche Weise wirksam ist.

Bleibt zu erweisen, daß es die Freimaurerei ist, die als Träger dieses kulturellen Unbewußten fungiert, indem, die Hieroglyphen der drey untern Grade das Vehiculum' dieser Weisheit, seyn, daß sie aber nichts destoweniger nie der Gegenstand des Suchens unserer Brüder werden könne oder müßte. ${ }^{35}$ Warum haben die ägyptischen Priester ihre Weisheit geheim gehalten? ,Edelmüthige und tugendhafte Menschen sind nie allein weise..., sondern setzen...ihre ganze Glückseligkeit darein,' ihr Wissen zum Wohle der Menschheit zum Tragen bringen zu können.

Wenn also Weise auf der Stuffe ihrer Vollendung Kenntnisse geheim halten, so ist kein anderer Beweggrund denkbar: als weil ihr Wissen solche Kenntnisse enthielt, die entweder den Profanen schädlich [werden] oder von ihnen... mißbraucht werden könnten, oder solche die das Volk über Dinge aufklären könnten, die es ihm besser ist, nicht zu wissen. ${ }^{36}$

Im Willen aber, diese Kenntnisse trotzdem zu überliefen, zielten die Ägypter über ihre eigene Gesellschaft hinaus und faßten die gesamte

\footnotetext{
${ }^{34}$ Kreil, ,Über die wissenschaftliche Maurerey', $65 f$.

${ }^{35}$ Ders., 66.

${ }^{36}$ Ders., 68.
} 
Menschheit in den Blick, ,denn sie baueten nicht für ihr Zeitalter, für ihre Nation, sondern für Jahrtausende, für ihre Gattung. ${ }^{37}$

Hinter diesem freimaurerischen Konzept der altägyptischen Geheimniskultur stand aber noch mehr als die platonische Idee von der Verborgenheit oder Schwerzugänglichkeit der Wahrheit, die nur der Philosoph bzw. der Eingeweihte unmittelbar anzustreben vermag, die dem gewöhnlichen Volk aber nur in Mythen, Allegorien und Bildern vermittelt werden kann. Die Freimaurer gaben der Spaltung der Kultur in Esoterik und Exoterik eine politische Deutung, von der die Griechen noch nichts ahnten.

Die maßgebliche Theorie entwickelte der englische Bischof, Literaturwissenschaftler und Altphilologe William Warburton in seinem Werk The Divine Legation of Moses, das 1738-1741 in drei Bänden erschienen war und in seinem zweiten von neun Büchern die antiken Mysterien behandelt. ${ }^{38}$ Die Heiden, die von der Offenbarung ausgeschlossen sind, waren-um Warburtons gewundenen Argumentationsgang kurz zusammenzufassen - auf das angewiesen, was man im 18. Jahrhundert „natürliche Theologie" nannte: die Erkenntnis Gottes aus der Natur, durch Rückschluss von der Schöpfung auf den Schöpfer. So wurden sie zu Deisten, ja Spinozisten avant la lettre. Auf dieser Religion aber, das war Warburtons These, läßt sich kein Staat aufbauen. Die Religion der Vernunft oder die natürliche Theologie kann nicht staatstragend sein. Der Staat braucht Götter zum Schutz der Gesetze und zum Ausdruck nationaler Identität. Die Elite schafft sich in der Form einer inneren Emigration die Mysterien, um in deren Schutz die Wahrheit über den fiktiven Charakter der politischen Götterwelt und die All-Einheit des Göttlichen zu bewahren und weiterzugeben.

Ohne die Furcht vor lohnenden und strafenden Gottheiten würden die Menschen die Gesetze nicht achten, und ohne Stadt-, Landes-, Staats- und Reichsgötter würde niemand wissen, was er ist und wohin er gehört. So kommt es zur Spaltung der Religion in einen exoterischen, staatstragenden Volkspolytheismus und einen esoterischen Deismus der Elite. Für diese doppelte, in eine exoterische und eine esoterische Seite gespaltene Philosophie oder Religion bot Warburton

${ }^{37}$ Ders., 69.

38 Warburton, The divine legation of Moses. 
nun eine politische Deutung an. Die esoterische Religion bzw. Philosophie musste im Verborgenen, im Untergrund praktiziert und tradiert werden, weil der fiktive Charakter der polytheistischen Götterwelt vor dem Volk geheim gehalten werden mußte. Warburton gab sich allerdings alle Mühe, die Alten Ägypter und die nach ihrem Modell eingerichteten heidnischen Staaten der Antike vor dem Vorwurf des Priesterbetrugs in Schutz zu nehmen, indem er die Unabdingbarkeit, ja, man ist im Vorgriff auf Nietzsche versucht zu sagen: die Lebensdienlichkeit der Fiktionen betont, auf denen die Volksreligion beruht. Ohne sie wären eine zivile Gesellschaft und ein geordnetes Staatswesen undenkbar. Man muss sie daher vor den Wahrheiten schützen, die sie als Fiktionen entlarven würden. Das setzte aber voraus, daß auch die eigentliche Wahrheit nicht vergessen, sondern von auserwählten Weisen im Untergrund gepflegt wurde. Ihren prominentesten Ausdruck fand diese Ägypten-Phantasie in Schillers Essay Die Sendung Moses (1789/90), der auf einem Traktat des befreundeten Illuminaten und Philosophen Carl Leonhard Reinhold über die ,Hebräischen Mysterien oder Die älteste religiöse Freymaurerey' basierte. ${ }^{39}$

Reinhold deutet die Hebräischen Mysterien als die ,älteste religiöse Freymaurerey', wobei mit Freymaurerey soviel wie Esoterik gemeint ist. Was aber bedeutet in diesem Zusammenhang "religiöse " Freimaurerei? Diesen Begriff versteht man nur, wenn man seinen Gegensatz kennt. Er heißt „wissenschaftliche Freimaurerei“. Der Erfinder dieser Unterscheidung und Terminologie ist kein anderer als jener Anton Kreil, den wir schon als den Autor jener Vorträge über das unterirdische Ägypten kennen gelernt haben, denen auch Mozart beiwohnte und die diesen, wie zu vermuten steht, für sein Projekt inspiriert haben, das Einweihungsritual der Pyramiden auf die Bühne zu bringen. Kreil und Reinhold waren beide Mitglieder der Loge zur Wahren Eintracht, und ihre Schriften über die ägyptischen und die hebräischen Mysterien stehen im Zusammenhang eines großangelegten Projekts dieser Loge zur Erforschung aller antiken Mysterien. Nicht weniger als 14 zum Teil monographisch angelegte Studien zu den ägyptischen, kabirischen, phönizischen, samothrakischen, eleusinischen, dionysischen, pythagoräischen, mithräischen, indischen, hermetischen, neuplatoni-

${ }^{39}$ Reinhold, Die hebräischen Mysterien. Dort ist auch Schillers Essay mit abgedruckt. 
schen Mysterien hatten Mitglieder dieser Loge erarbeitet und in ihrem Journal für Freimaurer veröffentlicht:

1. Ignaz von Born, Die Mysterien der Aegyptier; ${ }^{40}$

2. Karl Josef Michaeler, Ueber Analogie zwischen dem Christenthume der erstern Zeiten und der Freymaurerey; ${ }^{41}$

3. Joseph Anton von Bianchi, Über die Magie der alten Perser und die Mithrischen Geheimnisse; ${ }^{42}$

4. Ignaz von Born, Über die Mysterien der Indier; ${ }^{43}$

5. Anton Kreil, Geschichte des pythagoräischen Bundes; ${ }^{44}$

6. Karl Haidinger, Über die Magie; ${ }^{45}$

7. Anton Kreil, Geschichte der Neuplatoniker; ${ }^{46}$

8. Carl Leonhard Reinhold, Ueber die kabirischen Mysterien; ${ }^{47}$

9. [Anton Kreil], Über die wissenschaftliche Maurerey; ${ }^{48}$

10. Carl Leonhard Reinhold, Über die Mysterien der alten Hebräer; ${ }^{49}$

11. Augustin Veit von Schittlersberg, Ueber den Einfluß der Mysterien der Alten auf den Flor der Nationen; ${ }^{50}$

12. Anton Kreil, Ueber die eleusinischen Mysterien; ${ }^{51}$

13. Carl Leonhard Reinhold, Ueber die größern Mysterien der Hebräer; ${ }^{52}$

14. Michael Durdon, Über die Mysterien der Etrusker, insonderheit über die Geheimnisse des Bachus. ${ }^{53}$

Diese Loge hatte sich der "wissenschaftlichen Freimaurerei“ verschrieben und erblickte ihr Vorbild, vielleicht sogar ihren Ursprung, in den Priesterorden der Alten Ägypter, die, wie Anton Kreil und Ignaz von Born berichteten, ihren naturwissenschaftlichen, philosophischen und

\footnotetext{
40 Journal für Freymaurer 1, 1784, 15-132.

${ }^{41}$ Journal für Freymaurer 2, 1784, 5-63.

${ }^{42}$ Journal für Freymaurer 3, 1784, 5-96.

${ }^{43}$ Journal für Freymaurer 4, 1784, 5-54.

${ }^{44}$ Journal für Freymaurer 5, 1785, 3-28.

${ }^{45}$ Journal für Freymaurer 5, 1785, 29-56.

46 Journal für Freymaurer 6, 1785, 5-51.

47 Journal für Freymaurer 7, 1785, 5-48.

48 Journal für Freymaurer 7, 1985, 49-78.

49 Journal für Freymaurer 9, 1786, 5-79.

50 Journal für Freymaurer 9, 1786, 80-116.

51 Journal für Freymaurer 10, 1786, 5-42.

52 Journal für Freymaurer 11, 1786, 5-98.

53 Journal für Freymaurer 12, 1787, 5-164.
} 
theologischen Forschungen in unterirdischen Bibliotheken, Laboratorien, Forschungs- und Kultstätten nachgingen. So wie Ägypten die Heimstätte der wissenschaftlichen, so galt Israel als der Ursprung der religiösen Freimaurerei. Was hat man sich aber nun unter "religiöser Freymaurerey" vorzustellen? Reinhold veranschaulicht das anhand der israelitischen „Prophetenschule“. So wie die ägyptischen Eingeweihten die Vorläufer der „wissenschaftlichen Freimaurerei“, so waren die israelitischen Institutionen des Sanhedrin und besonders der „Prophetenschule" die Vorläufer der "religiösen Freimaurerei". ${ }^{54}$ Dieser Begriff ist also polemisch gemeint. Er bezeichnet das Verfallsstadium der Geheimniskultur. Solange nur die höchsten Eingeweihten noch eine gewisse Ahnung der Wahrheit haben, solange noch die komplexen hierarchischen und hieroglyphischen Architekturen der doppelten Religion auf den Fluchtpunkt der All-Einen Gottheit bezogen bleiben, ist gegen diese Ordnung nichts einzuwenden. Schlimm jedoch ist es, wenn der Schlüssel verloren geht, wenn im innersten Zentrum der Mysterien eine Leere herrscht, und wenn es nichts als diese Leere ist, die unter allen Umständen geheim gehalten werden muß. Dann verfallen die Mysterien zur bloßen Geheimniskrämerei und die Religion zum blinden Glauben. Mit diesen Warnungen wendet sich Reinhold am Schluß seines Buches wieder an die Freimaurerei zurück. Als Vertreter einer, eigentlichen und wahren Prophetenschule der Freymaurerey nennt er die "Strikte Observanz", den „Heiligen Orden des Gold- und Rosenkreuzes“ und das „Klerikat" des Orientalisten und Theologen Johann August Starck. Sie bilden die Front der religiösen Freimaurerei, das heißt einer leeren Geheimniskrämerei, der er das Programm der Illuminaten entgegenhält: die Umwandlung blinden Glaubens in ein Organ der Vernunft und letztendlich die Umwandlung der Gesellschaft in eine autonome Gemeinschaft mündiger Bürger, die Staat, Polizei, Kontrolle und Zensur nicht mehr nötig hat. Reinhold und seine Wiener Logenbrüder unterscheiden also zwischen zwei Formen von Esoterik, religiöser und wissenschaftlicher. Die eine kritisieren sie als leere Geheimniskrämerei und Aberglauben, die andere aber, die sie auf das Alte Ägypten zurückführen, nehmen sie sich zum Vorbild. Hier geht es um eine Wahrheit, die geheim bleiben muß, weil sie nicht staatstragend ist und die Fiktionen zerstören würde, auf die ein gut funktionierendes Staatswesen nun einmal angewiesen ist.

${ }^{54}$ te Lindert, ,Aufklärung und Heilserwartung`, 207. 
Diese Idee einer doppelten Kultur, die in zwei Ordnungen lebt, in der Ordnung der staatstragenden Fiktionen und in der Ordnung der universalen Wahrheit, findet sich nun auch außerhalb der im engeren Sinne esoterischen Kreise der europäischen Geheimgesellschaften. Besonders eindrucksvoll hat sie der jüdische Philosoph Moses Mendelssohn in seiner Schrift Jerusalem oder Religiöse Macht und Judentum entwickelt. ${ }^{55}$ Mendelssohn unterscheidet hier zwischen konkreten Religionen wie Judentum und Christentum einerseits, und, allgemeiner Menschenreligion' andererseits, also zwischen Glaubenswahrheiten und universalen Wahrheiten. Universale Wahrheiten (Mendelssohn nennt sie ,allgemeine Vernunftsätze') das ist seine These, sind den Juden nicht offenbart worden.

Diese offenbart der Ewige uns, wie allen übrigen Menschen, allezeit durch Natur und Sache, nie durch Wort und Schriftzeichen. (...) Sie wurden dem lebendigen, geistigen Unterrichte anvertrauet, der mit allen Veränderungen der Zeiten und Umstände gleichen Schritt hält. (...). Das Judentum wisse von keiner geoffenbarten Religion. Die Israeliten haben...Gesetze, Gebote, Lebensregeln, Unterricht vom Willen Gottes..., aber keine Lehrmeinungen, keine Heilswahrheiten, keine allgemeinen Vernunftsätze. Dieses ist allgemeine Menschenreligion, nicht Judentum; und allgemeine Menschenreligion, ohne welche die Menschen weder tugendhaft noch glückselig werden können, sollte hier nicht geoffenbart werden. ${ }^{56}$

Es gibt also im Judentum, und so sollte es nach Mendelssohns Ansicht auch in anderen Religionen sein, zwei normative Orientierungen: einmal an den Glaubenswahrheiten der konkreten Religion, wie sie in heiligen Schriften niedergelegt sind, und zum anderen an den „universalen Vernunftsätzen“ der allgemeinen Menschenreligion, wie sie niemals festgeschrieben, sondern immer nur diskursiv, ,mit allen Veränderungen der Zeiten und Umstände gleichen Schritt' haltend, ausgehandelt werden können. Die universalen Wahrheiten der allgemeinen Menschenreligion entsprechen dem, was nach Vorstellung der Wiener Freimaurer die ägyptischen Priester im Untergrund betrieben haben und was auch jetzt, im aufgeklärten Zeitalter des 18. Jahrhunderts, die Freimaurer noch zwingt, im Schutzraum des Logengeheimnisses zu verhandeln. Auch der aufgeklärte Absolutismus des 18. Jahrhunderts, in Preußen, Österreich und Frankreich, war noch eine

\footnotetext{
${ }^{55}$,Mendessohn, Jerusalem:

${ }^{56}$ Ders., $407 f$.
} 
Geheimniskultur und die Aufklärung war noch immer eng mit der Esoterik verschwistert, wie besonders die Forschungen von Monika Neugebauer-Wölk und ihres Kreises deutlich gemacht haben. Doch bietet sich in der Kunst ein Raum, die Geheimnisse und Grundsätze der allgemeinen Menschenreligion nach außen, zumindest auf die Bühne zu tragen. Künstler-Freimaurer, wie Lessing mit Nathan der Weise, Mozart mit der Zauberflöte, Goethe und Herder mit verschiedenen ihrer Werke sind darin am weitesten gegangen. Schiller, der zwar kein Freimaurer war, aber durch seine Freundschaft mit Reinhold intime Kenntnisse der Geheimbünde besaß, hat in Marquis Posa das Idealportrait eines Illuminaten gezeichnet. Mit Recht wird die Erklärung der Menschenrechte in den USA 1776 mit diesen Bestrebungen in Verbindung gebracht. Die Französische Revolution hat dann versucht, die traditionelle Religion abzuschaffen und durch eine besondere Fassung der allgemeinen Menschenreligion, den Kult des Höchsten Wesens und der Vernunft zu ersetzen. Die bürgerlichdemokratische Gesellschaft des 19. und 20. Jahrhunderts bedeutete das Ende der doppelten Kultur. Zwar blühten verschiedene Geheimbünde und Formen von Esoterik weiterhin, aber sie verloren ihren Bezug zu Aufklärung und Universalismus und etablierten sich in der genauen Gegenrichtung. Das bürgerliche Zeitalter bedeutete das Endezwar nicht der Esoterik, aber ihres kulturellen und gesellschaftlichen Stellenwertes.

\section{Literaturverzeichnis}

,Philippus-Evangelium', in: Werner Foerster (Hg.), Die Gnosis. Koptische und mandäische Quellen. Bd 2, Zürich: Artemis 1971, 108.

Ammianus Marcellinus = Ammien Marcellin, Histoire, Tome III: livres xx-xxii, übers. von J. Fontaine (Hg.), Paris 1996.

Anonymus, Athenian letters or, the Epistolary Correspondence of an Agent of the King of Persia, residing at Athens during the Peloponnesian war. Containing the History of the Times, in Dispatches to the Ministers of State at the Persian Court. Besides Letters on various subjects between Him and His Friends, Bd. 1. London: James Bettenham 1741.

Assmann, Aleida, Erinnerungsräume. Formen und Wandlungen des kulturellen Gedächtnisses, München: C. H. Beck 1999.

Assmann, Jan, ,Das gerettete Wissen. Flutkatastrophen und geheime Archive, in: Mulsow/Assmann (Hg.), Sintflut und Gedächtnis, Erinnern und Vergessen des Ursprungs, Paderborn: Fink 2006.

- , Die Zauberflöte. Oper und Mysterium, München: Hanser 2005.

_-, Antike Äußerungen zur ägyptischen Schrift', in: Aleida Assmann, Jan Assmann (Hg.), Hieroglyphen. Stationen einer anderen abendländischen Grammatologie, München: Fink 2003, 27-35. 
- ,Etymographie: Zeichen im Jenseits der Sprache‘, in: Aleida Assmann, Jan Assmann (Hg.), Hieroglyphen. Stationen einer anderen abendländischen Grammatologie, München: Fink 2003, 37-63.

-, ,Hieroglyphen: altägyptische Ursprünge abendländischer Grammatologie‘, in: Aleida Assmann, Jan Assmann (Hg.), Hieroglyphen. Stationen einer anderen abendländischen Grammatologie, München: Fink 2003, 9-25.

Buno, Johannes, and von Klingenberg, Paul, Historische Bilder/ Darinnen Idea Historiae Universalis: Eine kurtze Summarische Abbildung der Fürnehmsten Geist- und Weltichen Geschichte von Anfang der Welt durch die Patriarchen/ Richter und Könige in Israel und Juda/ die IV. Monarchien, sampt andern Königen. Wittenberg: Schultzische Schrifften, 1693.

Clemens Alexandrinus, Stromata Buch I-VI, hrsg. v. Otto Stählin, Berlin: AkademieVerlag, 4. Aufl. 1985.

Dozy, Reinhart und de Goeje, Michael Jan, Description de l'Afrique Texte arabe publié pour la première fois d'après les ms. de Paris et d'Oxford avec une trad., des notes et un glossaire. 2 Bd., Leiden: Brill 1866.

Eusebius, ,Die Praeparatio Evangelica', hrsg. v. Karl Mras, in: Eusebius. Werke, 8.1, GCS 43,1, Berlin: Akademie-Verlag 1982.

Ginzberg, Louis, Legends of the Jews (1909), Bd.I, Baltimore: John Hopkins UP 1998.

Goethe, Johann Wolfgang von, ,Aus Makariens Archiv', in: Erich Trunz (Hg.) Goethes Werke (Hamburger Ausgabe) 8, München: Beck 1981, 460 Nr. 3.

Hornung, Erik, Das esoterische Ägypten. Das geheime Wissen der Ägypter und sein Einfluss auf das Abendland, München: dtv 1999.

Journal für Freimaurer, Bände I-IV in 12 Teilbänden (alles erschienene), Wien: Christian Friedrich Wappler 1784-1787.

Kreil, Anton, ,Über die wissenschaftliche Maurerey', in: Journal für Freymaurer 7, Wien: $1785,49-78$.

Lindert, Wilgert te, ,Aufklärung und Heilserwartung. Philosophische und religiöse Ideen Wiener Freimaurer (1780-1795)', in: Reinalter Helmut (Hg.), Schriftenreihe der Internationalen Forschungsstelle Demokratische Bewegungen in Mitteleuropa 1770-1850. Bd. 26, Frankfurt a.M.: Peter Lang 1998, 207.

Mendessohn, Moses, ,Jerusalem oder Über religiöse Macht und Judentum', in: Martina Thom (Hg.), Schriften über Religion und Aufklärung, Darmstadt: Wiss. Buchges. 1989, 351-458.

Mulsow, Martin/ Assmann, Jan (Hg.), Sintflut und Gedächtnis. Erinnern und Vergessen des Ursprungs, Paderborn: Fink 2006.

Origenes, ,Contra Celsum' (cap. 12), in: Jacques Paul Migne (Hg.), Patrologia Graeca 11, Paris: Migne 1857.

Pauw, Cornelius de, Recherches philosophiques sur les Egyptiens et les Chinois, Berlin: G. J. Decker 1773.

Plutarch, De Iside et Osiride, Prag: Orientalisches Institut 1940/41.

Reinhold, Carl Leonhard, Die hebräischen Mysterien oder die älteste religiöse Freimaurerei, Leipzig: Göschen 1788; Neudr., hg. v. Jan Assmann, Neckargemünd: Edition Mnemosyne 2001 (2. Auflage 2006).

Schneider, Manfred, ,Liturgien der Erinnerung, Techniken des Vergessens', in: Merkur 41: 8 (1987), 676-686.

Thissen, Heinz Josef (Hg.), Des Niloten Horapollon Hieroglyphenbuch, München:Saur 2001.

Waddell, William Gillian (Hg.), Manetho. Werke. Cambridge, MA: Harvard University Press 1964.

Warburton, William, The Divine Legation of Moses demonstrated on the Principles of a Religious Deist, from the Omission of the Doctrine of a Future State of Reward and Punishment in the Jewish Dispensation. London: Millar, 1738-1741. 